\title{
COVID-19: Rescue by transcriptional inhibition
}

\author{
Ali Shilatifard \\ Editor, Science Advances; Chairman, Department of Biochemistry and Molecular Genetics; Director, Simpson Querrey Center for Epigenetics, Northwestern University \\ Feinberg School of Medicine, SQBRC 7-516, 330 E. Superior St., Chicago, IL 60611 USA. \\ Email: ASH@Northwestern.edu
}

The National Institutes of Health (NIH) recently disclosed preliminary results from their highly anticipated clinical study with the viral specific transcriptional inhibitor Remdesivir for the treatment of adults suffering from severe COVID-19. The results as indicated in their news release, are very encouraging! You may ask, why transcription, and how is transcription being targeted for the treatment of COVID19 ? Here is a short answer for you. The genetic information in our genome and that of all other living organisms is stored in the form of DNA. Viruses, however, are not living organisms but similarly store their genetic information in the form of nucleic acids as well, but either as DNA or RNA, depending on the virus.

Before the information in the genome can be translated into proteins, this genetic information needs to be transcribed to generate an intermediate molecule known as messenger ribonucleic acid (mRNA). The enzyme involved in the synthesis of RNA is RNA polymerase. RNA polymerases are highly conserved, both structurally and functionally from single-cell bacteria to humans, with the difference being that there are more RNA polymerase genes/subunits in the human genome and many auxiliary factors that regulate their function in the transcription of the densely packed human genome. This past fall, we celebrated the $50^{\text {th }}$ anniversary of the discovery of the RNA polymerase in eukaryotes, fantastic work done by biochemists Robert Roeder and Pierre Chambon. During the past five decades much has been learned, not only about the structure/function relationship of RNA polymerases, but also about the auxiliary factors that work with them to orchestrate the very complicated process of gene expression to create and sustain life.

Some viruses like the common cold, influenza, hepatitis C, SARS, Ebola and SARS-CoV2, the causative virus of COVID19 are RNA viruses, hence their genetic information is in the form of RNA. Since human cells don't contain the enzymes RNA viruses need to replicate their genome, we can target these enzymes when designing therapies for these infections. One such target is SARS-CoV2's RNA dependent polymerase (RdRp). Just like in other RNA viruses, the RdRp is indispensable for replicating the SARS-CoV-2 RNA genome as well as for generating additional RNAs via transcription to propagate more viruses. An indispensable feature for survival of RNA viruses is the error-prone nature of the $\mathrm{RdRp}$ in replication of the viral genome, which allows the adaptation and speedy evolution for the virus as well as generation of mutant versions of the virus that can be more infectious and harmful than the original one.

In an earlier attempt to develop therapeutic approaches for the treatment of Ebola, which also depends on RdRp for its propagation, Gilead Sciences developed "Remdesivir," an adenosine nucleotide analog chain terminator for RdRps, which can be incorporated into the nascent viral RNA and cause premature termination of viral-specific transcription. Although the clinical data for the treatment of Ebola with "Remdesivir" was not encouraging, recently, this drug was tested in a series of clinical trials in individuals suffering from severe COVID-19. Gilead recently announced encouraging results of their uncontrolled study with Remdesivir and on April 29, NIH shared preliminary results for their doubleblind, placebo-controlled, multi-center trial study. In both studies, therapy with Remdesivir resulted in a reduced time to recovery for hospitalized patients with advanced COVID19. In addition, patients on 10-day versus 5-day regimens of Remdesivir demonstrated similar positive outcomes, suggesting the efficacy of the drug in a shorter time of therapy. It is worth noting that a 10-hospital study report from Wuhan, China did not observe these benefits of Remdesivir. However, this trial was not as comprehensive and methodical as the NIH study and was underpowered, as it was terminated because of insufficient enrollment before reaching its pre-specified sample size.

These initial encouraging clinical studies from the United States with Remdesivir for the treatment of COVID-19 are very motivating as a source of hope. There is no doubt that many analogs of Remdesivir or other viral inhibitors such as EIDD-1931 or its clinical tool compound EIDD-2801 will soon be tested for their antiviral efficacy toward SARS-CoV-2 infection in human. These analogs may not only demonstrate great efficacy toward SARS-CoV-2 infection, but also allow scientists to tackle the problems of drug resistance associated with RNA viruses given their either high error-prone nature for some RdRp, or high replication rate of the viral genome as the result of a high level of infection. Additionally, the development of clinical regimens with such viral-specific inhibitors that can be synergistic with IL-6 blockade clinical tools being tested, may prove extremely successful for the 
treatment of individuals with severe COVID-19 presenting with cytokine storm and severe systemic inflammatory response.

There are not enough words to thank our clinical colleagues on the front lines of this battle, and their selfless and tireless contributions are to be extolled. Together with them, we should also commend the graduate students, postdocs, and scientists in academia and industry who spend seemingly infinite amounts of time measured in years and decades applying comprehensive, methodical, biochemical and molecular approaches to their studies. Without these kinds of heroic efforts over the last 50 years, we would lack the detailed molecular knowledge of transcription allowing the development of antiviral therapies for COVID-19 today. Using these approaches and working diligently and enduringly toward the generation of a vaccine and effective treatments to ward off this invisible enemy, we will be able to light the path out of this pandemic and away from any future ones.

Published First Release 11 May 2020

10.1126/sciadv.abc6891 